\title{
LAZY YET EFFICIENT LAND-COVER MAP GENERATION FOR HR OPTICAL IMAGES
}

\author{
J. Michel \\ C-S - IGI \\ Parc de la Plaine \\ Rue Brindejonc des moulinais, BP 5872 \\ 31506 TOULOUSE CEDEX 5 - France
}

\author{
J. Inglada \\ CNES - DCT/SI/AP - BPI 1219 \\ 18 avenue Edouard Belin \\ 31401 TOULOUSE CEDEX 9 - France
}

\section{INTRODUCTION}

The task of automatically or semi-automatically deriving land-cover and land-use maps is difficult to achieve in the context of high spatial resolution images. The level of details that can be observed as well as the diversity of objects in the images imply to define many classes. Moreover, the pixel or neighborhood based approaches are not able to capture the richness of the information, since objects are composed of one or several groups of radiometric-coherent pixels whose shape and extent can not be known a-priori. To overcome these drawbacks, a new set of techniques, called object-based image analysis (OBIA for short), has come to light. OBIA often implies a preliminary segmentation step in which image pixels are clustered into these radiometric-coherent groups of pixels. These so-called segments can then be classified in a supervised or unsupervised way using a variety of attributes describing them. This approach better suits high resolution imagery, but some issues are still not yet properly addressed. This paper aims at trying to overcome them by proposing a new scheme based on state-of-the-art tools from the literature.

\section{DRAWBACKS OF CLASSICAL OBJECT-BASED IMAGE ANALYSIS APPROACHES}

This section presents the main issues when trying to perform land-cover and land-use with classical object-based image analysis.

\subsection{Importance of preliminary segmentation}

The first major drawback of OBIA is that one has to rely on a preliminary segmentation. If algorithms like the Mean-Shift [1, 2], graph-cuts [3] or some optimized version of the watershed transform are very efficient to segment some predefined structures after parameters tuning, a good global segmentation segmenting each object of the image at its right scale (and thus avoiding over and under-segmentation) seems not achievable at all. Moreover, thin and long objects are often penalized by these methods designed for compact and homogeneous objects.

\subsection{Limited set of features}

The second drawback of these approaches is that they integrate only a limited set of features [4]. Geometry and radiometry are almost the only attributes that are available in market tools. If they allow to distinguish classes up to a certain point, they do not account for neighboring regions or textures, which are valuable information when dealing with high-resolution images. Moreover, geometry attributes such as Flusser moments [5] for instance are highly sensitive to segmentation errors, and therefore can not always be trusted.

\subsection{Intensive implication of the human operator}

In [6], authors point out that the use of existing OBIA approaches remain highly tedious in operational contexts : "Desired classifications are often reached only after extensive trial and error, which can be time consuming. Furthermore, object-oriented classification may require the interpreter to be familiar with the geographic area or object of interest prior to completing a classification". The human operator is responsible for almost everything : 
- Deciding the number and nomenclature of the classes, without sometimes knowing which classes the image(s) actually contain,

- Select training samples without actually knowing which samples are really important for the system to learn properly,

- Select meaningful features, and sometimes even their relative weights and decision thresholds, without knowing which ones do actually improve the system accuracy.

\section{THE PROPOSED APPROACH}

To overcome the limitations cited in section 2, we propose a new framework to produce land-use and land-cover maps, in which the set of features can be easily and unlimitedly extended, and the tedious part of selecting meaningful features and samples is automated as much as possible. The main steps of this framework are presented figure 1. Here is how it works. After the image has been segmented (see section 3.1), each segment is given a set of user-defined attributes (see section 3.2). To initialize the process, the user manually selects training samples corresponding to her classes of interest among the segments. She may also select the features she wants to focus on, even if it is not necessary that she perform the feature selection (see section 3.3). It then starts training an SVM classifier with these samples and these features. After the training process, which is believed to be rather short due to the small number of training samples (for a start, 10 samples per class might be sufficient), the framework proposes to the user a set of samples for which it is the less confident (see section 3.4). This process is also known as active learning [7]. The operator then chooses to label some of the proposed samples, or eventually labels other samples that were not proposed, and iterates through this process until a satisfactory level of classification is reached. The classifier parameters (i.e. support vectors) can then be exported and applied to other images as well. As a matter of fact, it would be possible to apply this framework to the joint production of several land-cover or land-use maps from several input images. Figure 3 shows results after a few iterations of this approach applied to a pan-sharpened Quickbird patch.

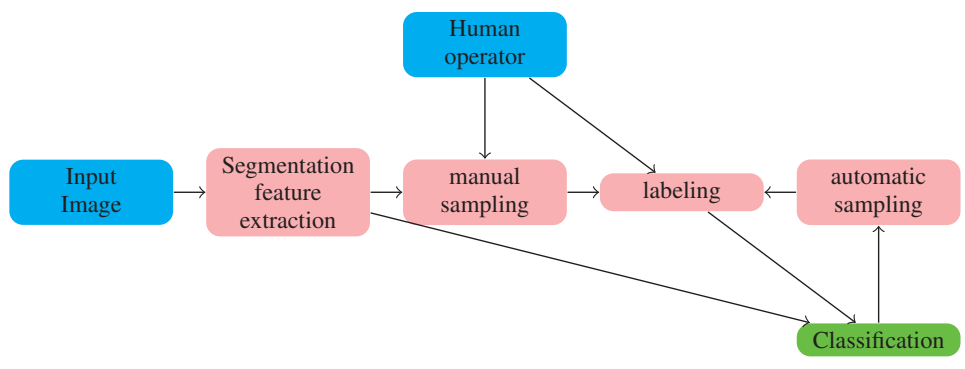

Fig. 1. Block diagram of the proposed approach

\subsection{Segmentation}

In previous works [8], we have used morphological approaches for image segmentation [9]. Although the obtained results are satisfactory, it is not straightforward to integrate the full spectral content of remote sensing images in this framework. Therefore, we decided to use the Mean-Shift algorithm [1], which is able to integrate multi-spectral data without further modification or pre-processing and whose parameters are simple to set. However, with this choice we loose the multi-scale capabilities of the morphological profiles.

\subsection{Generic features}

Our framework allows the use of any kind of feature which can be computed for a region of an image yielded by a previous segmentation. We currently use the following, although others can be included without limitation:

- geometric attributes: Flusser moments, elongation, physical size;

- radiometric attributes: vegetation indices, water indices, redness, brightness;

- statistics and textures: mean,variance, kurtosis, skewness; 


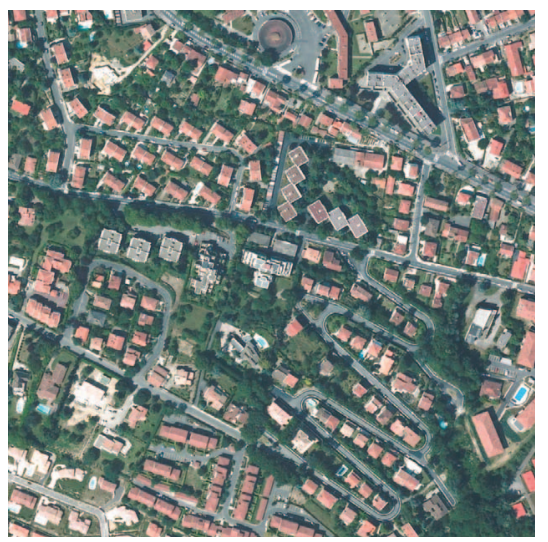

(a) Input image

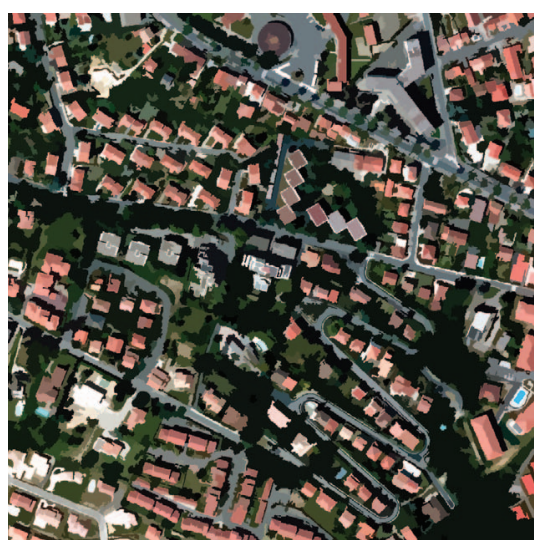

(b) Clustered image

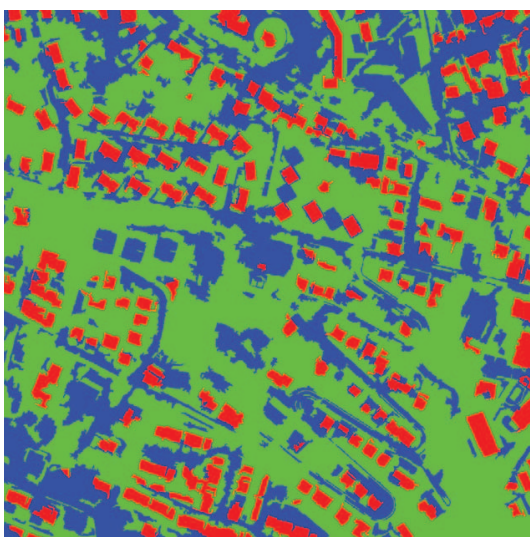

(c) Classification

Fig. 2. Results obtained after a few iterations on a pan-sharpened Quickbird patch with less than 10 examples per class

- contextual attributes: bag of words of neighboring regions.

We plan to include also spatial reasoning relationships as we did in [8].

\subsection{Feature selection}

Since the number of features used by the system may be overwhelming (see section 3.2), the issue of feature selection has to be addressed. Our classification system is based on Support Vector Machines, so it is rather robust to the dimensionality if the feature space. Nevertheless, the user has the possibility of removing features.

No automatic feature selection scheme is implemented in the system, since the obtained results have been satisfactory so far, but techniques as those assessed in [10] can be included in the system easily.

\subsection{Samples selection}

The key point for a lazy yet efficient supervised machine learning framework is the selection of the most pertinent samples so their amount can be reduced, thus allowing for a faster learning phase.

SVM, by construction, select the most significant samples: these are the support vectors. This step is performed during learning, which means that the support vectors will be a subset of the samples selected by the operator.

However, how to previously select samples so they allow an efficient learning?

Active learning approaches use the classifier itself in order to select the most meaningful samples for the learning. We have implemented a simple approach suited for SVM classifiers: the margin sampling selection [7]. It uses the fact that samples which are close to class boundaries are the most useful for an SVM classifier. Therefore, the classifier selects non-labeled regions which lay inside the margin areas of the feature space and presents them to the user for decision.

\subsection{Class discovery}

One interesting characteristic of active learning approaches is that often, the classifier will select for the user to decide on non-labeled objects which do not belong to any of the classes of the current nomenclature.

The user has at least 3 options:

1. she decides that the object is not interesting and therefore it belongs to the "reject" class;

2. she decides that it is interesting and creates the appropriate class;

3. she decides that the object may be interesting, but she is unable to assign it to a class and she can put it in the "don't know class". 
Another interesting phenomenon occurs if we allow the sample margin algorithm to select already labeled classes. This allows the classifier to identify objects difficult to class and therefore gives the opportunity to the user to relabel the object by assigning it to an existing or to a newly created class.

\section{LIMITATIONS}

Although successful in terms of results, our system still needs improvements. We have identified a set of items on which we are right now working or plan to work in the near future:

1. need for an existing segmentation;

2. scale aspect of segmentation;

3. automatic feature selection scheme;

4. way contextual information is accounted for.

These items will be discussed in the final paper.

\section{LIVE DEMONSTRATION}

This framework was implemented in a proof-of-concept application based on the Orfeo ToolBox (www. orfeo-toolbox. org). Hence, the final presentation will include a live demonstration of the capabilities of the framework.

\section{REFERENCES}

[1] D. Commaniciu, "Mean Shift: A Robust Approach Toward Feature Space Analysis," IEEE Transactions on Pattern Analysis and Machine Intelligence, vol. 24, no. 5, pp. 603-619, May 2002.

[2] "Edison: Code for the edge detection and image segmentation," http://www.caip.rutgers.edu/riul/research/code/EDISON.

[3] Yuri Boykov and Gareth Funka-Lea, "Graph cuts and efficient n-d image segmentation," International Journal of Computer Vision, vol. 70, no. 2, pp. 109-131, Nov. 2006.

[4] Sven Nussbaum and Gunter Menz, Object-Based Image Analysis and Treaty Verification New Approaches in Remote Sensing Applied to Nuclear Facilities in Iran.

[5] Jan Flusser, "On the independence of rotation moment invariants," Pattern Recognition, vol. 33, pp. 1405-1410, 2000.

[6] Hugh Devine Justin M. Shedd and Dan Hurlbert, Mapping forest hurricane damage using automated feature extraction.

[7] Greg Schohn and David Cohn, "Less is more: Active learning with support vector machines," in ICML '00: Proceedings of the Seventeenth International Conference on Machine Learning, San Francisco, CA, USA, 2000, pp. 839-846, Morgan Kaufmann Publishers Inc.

[8] J. Inglada and J. Michel, "Qualitative spatial reasoning for high-resolution remote sensing image analysis," IEEE Transactions on Geoscience and Remote Sensing, vol. 47, no. 2, pp. 599-612, Feb. 2009.

[9] Martino Pesaresi and Jon Atli Benediktsson, "A new approach for the morphological segmentation of high-resolution satellite imagery," IEEE Transactions on Geoscience and Remote Sensing, vol. 39, no. 2, pp. 309-320, february 2001.

[10] T. Habib, J. Inglada, G. Mercier, and J. Chanussot, "Assessment of feature selection techniques for support vector machine classification of satellite imagery," in IEEE International Geoscience and Remote Sensing Symposium, 2008, 2008, vol. 4, pp. 85-88. 